\title{
Innovative and Emerging Drying Technologies for Enhancing Food Quality
}

\author{
Hong-Wei Xiao ${ }^{1},{ }^{1}$ Zhongli Pan, ${ }^{2,3}$ Alex Martynenko, ${ }^{4}$ Chung-Lim Law, ${ }^{5}$ \\ and Prabhat K. Nema ${ }^{6}$ \\ ${ }^{1}$ College of Engineering, China Agricultural University, Beijing, China \\ ${ }^{2}$ Western Regional Research Center, USDA-ARS, Albany, CA, USA \\ ${ }^{3}$ University of California, Davis, CA, USA \\ ${ }^{4}$ Dalhousie University, Halifax, NS, Canada \\ ${ }^{5}$ Department of Chemical and Environmental Engineering, Faculty of Engineering, The University of Nottingham, \\ Malaysia Campus, Jalan Broga, Semenyih, Selangor Darul Ehsan, Malaysia \\ ${ }^{6}$ Department of Food Engineering, National Institute of Food Technology Entrepreneurship and Management, Kundli, Sonepat, \\ Haryana 131028, India
}

Correspondence should be addressed to Hong-Wei Xiao; xhwcaugxy@163.com

Received 10 April 2018; Accepted 10 April 2018; Published 13 May 2018

Copyright (c) 2018 Hong-Wei Xiao et al. This is an open access article distributed under the Creative Commons Attribution License, which permits unrestricted use, distribution, and reproduction in any medium, provided the original work is properly cited.

Drying is one of the most frequently used methods for food preservation due to prevention of microorganisms' growth and reduction of moisture-mediated deteriorative reactions at low water activity. In addition, food drying provides numerous benefits, such as formation of desirable texture and physical properties, extending shelf life, and minimizing packaging, storage, and transportation costs. Although simplicity and small capital investments make natural opensun drying and hot air convective drying the most popular practical technologies for drying of agricultural products in developing countries, food quality remains the major issue. For example, open-sun drying has several disadvantages, including long drying time, rewetting or rotting caused by bad weather, contamination by dust and insects, nonuniform drying, and significant color loss and nutrients deterioration due to long exposure to solar radiation. Drawbacks of hot air drying include degradation in nutrients and flavor, shrinkage, fractures and case hardening, color darkening, and decrease of antioxidant and rehydration capacity due to prolonged exposure to high air temperatures. These technologies require critical improvements to improve the product quality and processing sustainability.

With globalization of world economy, food quality became an industry priority since it provides companies competitive advantage in the global market place. Therefore, recent trend in the food drying research focuses on minimizing chemical and thermal degradation and maximizing nutrient retention in food products. Innovative and emerging drying technologies offer key advantages to improve food quality and address growing consumer demand on the global food market. In recent years, a range of novel drying technologies has been developed to improve physicochemical properties of foods by minimizing thermal degradation and time of drying. The primary effort of these innovations is to increase drying efficiency with minimal changes in nutritional value of foods and extension of shelf stability to ensure product safety, quality, and acceptability. Another aspect of innovations is to minimize energy consumption and reduce carbon footprint of drying technologies.

The main goal of this special issue is to provide a platform for the discussion on the latest progress of innovative and emerging drying technologies. Following a call for papers, more than 14 manuscripts were submitted for this special issue, and after being strictly peer reviewed, 8 manuscripts were finally accepted.

Fruit and vegetable chips are very popular with consumers due to its desired texture. The research work from 
Min Xiao and corroborators verified that the modifications of pectic polysaccharides of apple chips during drying significantly affect the texture of apple chips. Vacuum frying is a recently developed technique for chips processing with low oil content. The investigation from Aiqing Ren and collaborators focused on using pretreatment to preserve nutrients and reduce oil content of vacuum-fried shiitake mushroom slice. They reported the blanching, osmotic dehydration, and coating pretreatment before vacuum frying could improve color and sensory evaluation and minimize the oil uptake of vacuum-fried shiitake mushroom chips. To avoid intake of excessive energy during consumption of fried chips, the research work from Dr. Jiangyong $\mathrm{Yi}$ and collaborators applied a combined freeze drying and instant controlled pressure drop process (FD-DIC) on carrot-potato chips processing, verified that FD-DIC is an alternative method for obtaining desirable restructured fruit and vegetable chips, and optimized the processing conditions of carrot-potato chips by using response surface methodology.

Microencapsulation is widely used to minimize the oxidation of fish oil products. The study from Yuqi Pang and coworkers compared the effects of different drying methods (spray drying, freeze drying, and spray freeze drying) on the microencapsulation of fish oil. The authors found that spray freeze drying obtained high quality and more stability powder, which is a promising method for the preparation of microcapsules, which can improve product storage stability and potential digestibility.

Sun drying and convective drying are still the most frequently used drying methods in many developing countries. The paper of Iva Kučerová and coworkers applied a double-pass solar drier for thin-layer drying of eland and beef, when combined with pretreatment of traditional jerky marinade with fresh pineapple juice, obtained a favorable texture, color, and taste product. The work from Roberto Lemus-Mondaca reported that the hot air drying process is an important processing of abalone, air temperature significantly influenced the physical, chemical, and nutritional properties of osmo-treated abalone slices, discoloration of product was more evident at high drying temperatures, and the drying temperature of $60^{\circ} \mathrm{C}$ could enhance the final characteristics of the product.

Drying is a complex, dynamic, highly nonlinear, strongly interactive, and multivariable thermal process. The prediction of moisture content and quality parameters is very useful to improve the overall performance of drying process. Artificial neural network (ANN) methodology could precisely predict experimental data. The paper from Dr. JunWen Bai and coworkers modelled the experimental drying kinetics and color changes of ginkgo biloba seeds during its microwave drying process using ANN methodology. The authors verified that ANN methodology could precisely predict experimental data; meanwhile, the established ANN models could be used for online prediction of moisture content and color changes of ginkgo biloba seeds during microwave drying process.

Blanching is an important pretreatment prior to agroproducts drying, to inactivate enzymes and remove air from intercellular space to prevent color and flavor degradation during drying. The paper of Hai-ou Wang and coworkers evaluated the effect of hot water blanching on the drying characteristics and product qualities of apple slices dried with the novel integrated freeze-drying process. It was found that hot water blanching pretreatment is a promising alternative method to decrease drying time and improve product quality of apple slices.

Finally, the research work from Dr. Zhijun Zhang and collaborators revealed the shape effect on the temperature field during microwave heating. They reported that, for the small and big volume samples, spherical sample was better than the cubic ones, and for the intermediate size samples, temperature uniformity of spherical sample is better than that of cubic sample, while the microwave absorption capability of cubic sample is higher than that of spherical sample. Besides, the results also indicated that orientations affect the microwave process significantly. Therefore, an optimal sample shape and orientation could be decided to achieve the goal of high temperature distribution uniformity and low energy consumption.

We hope that this special issue not only contributes to a better understanding of the research status of innovative and emerging drying techniques, but also triggers new research opportunities in this field in order to provide more healthy and nutritious food for the growing global population in a more sustainable way. We hope that the readers will enjoy the reading and this publication will inspire further investigation in this exciting and promising research area.

\section{Hong-Wei Xiao Zhongli Pan \\ Alex Martynenko Chung-Lim Law \\ Prabhat K. Nema}

\section{Acknowledgments}

The authors would like to sincerely thank all the authors who contributed to this special issue, as well as the expert reviewers who provided constructive feedback and extremely useful comments. 


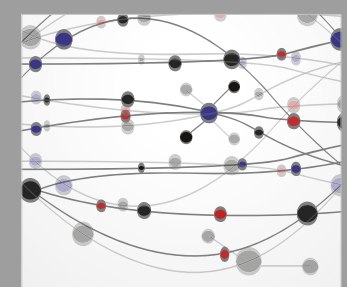

The Scientific World Journal
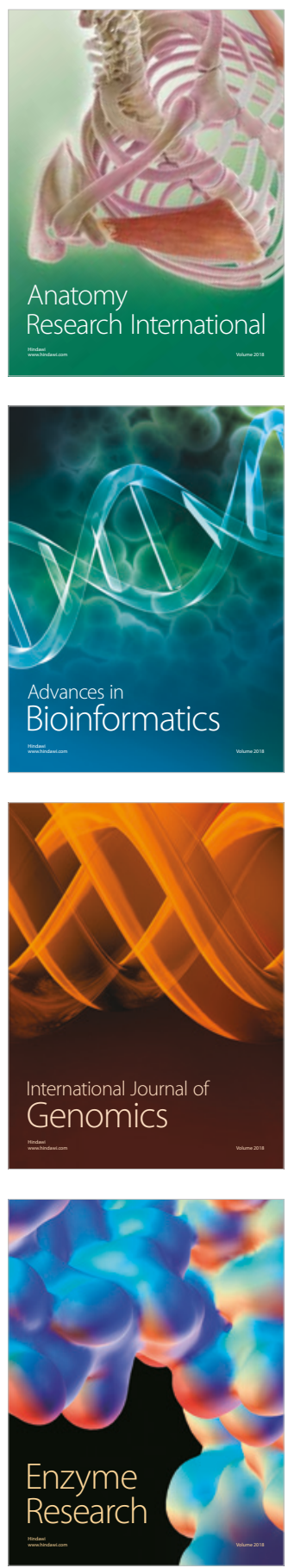
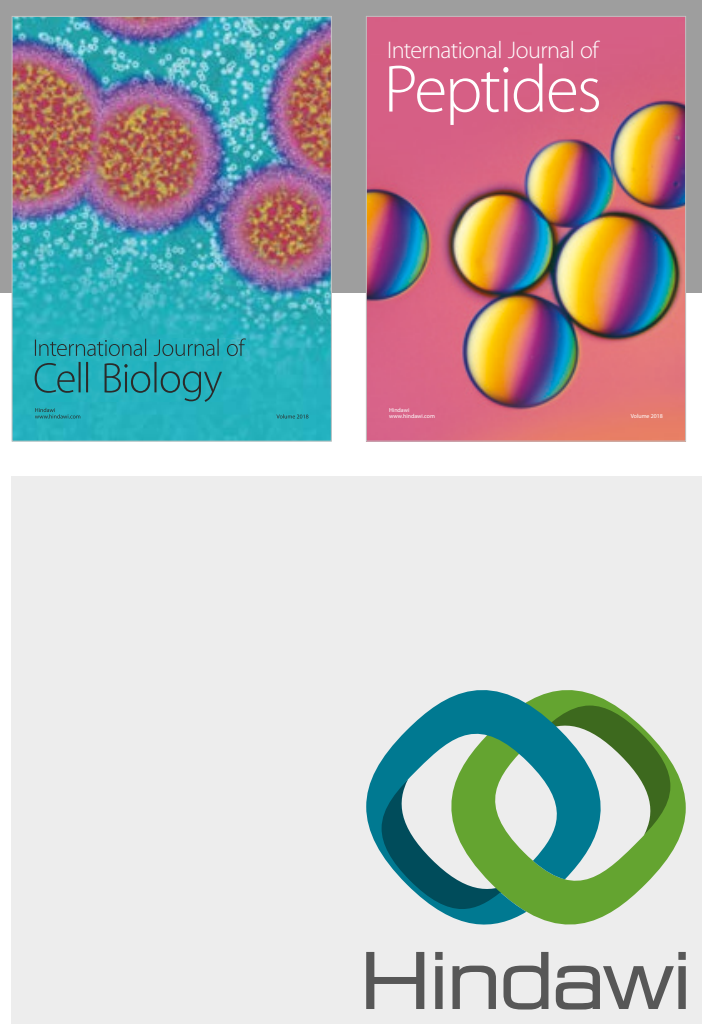

Submit your manuscripts at

www.hindawi.com
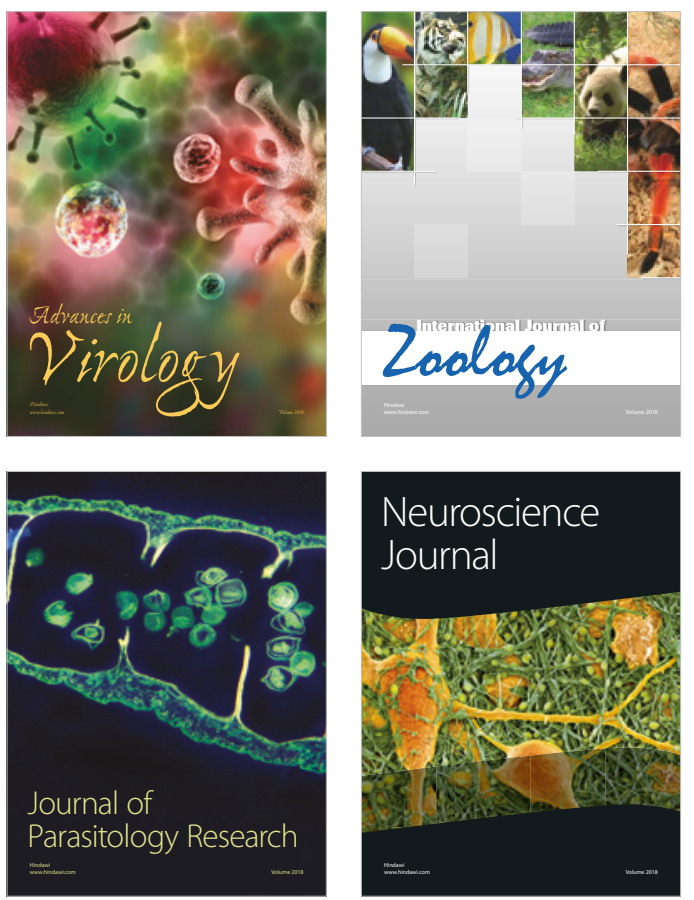
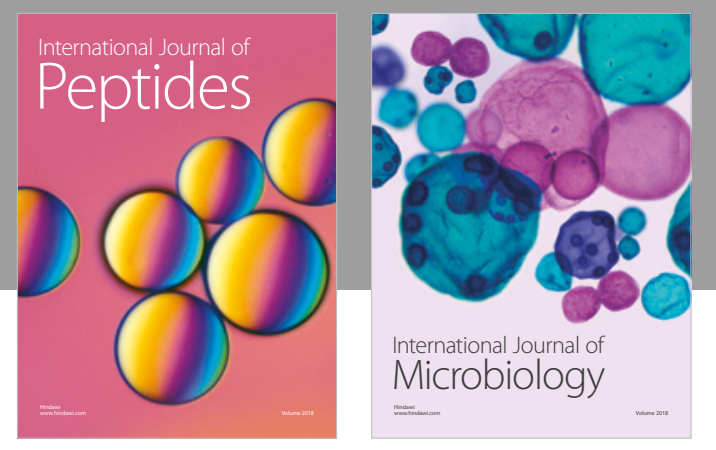

nternational Journal of Microbiology
Journal of
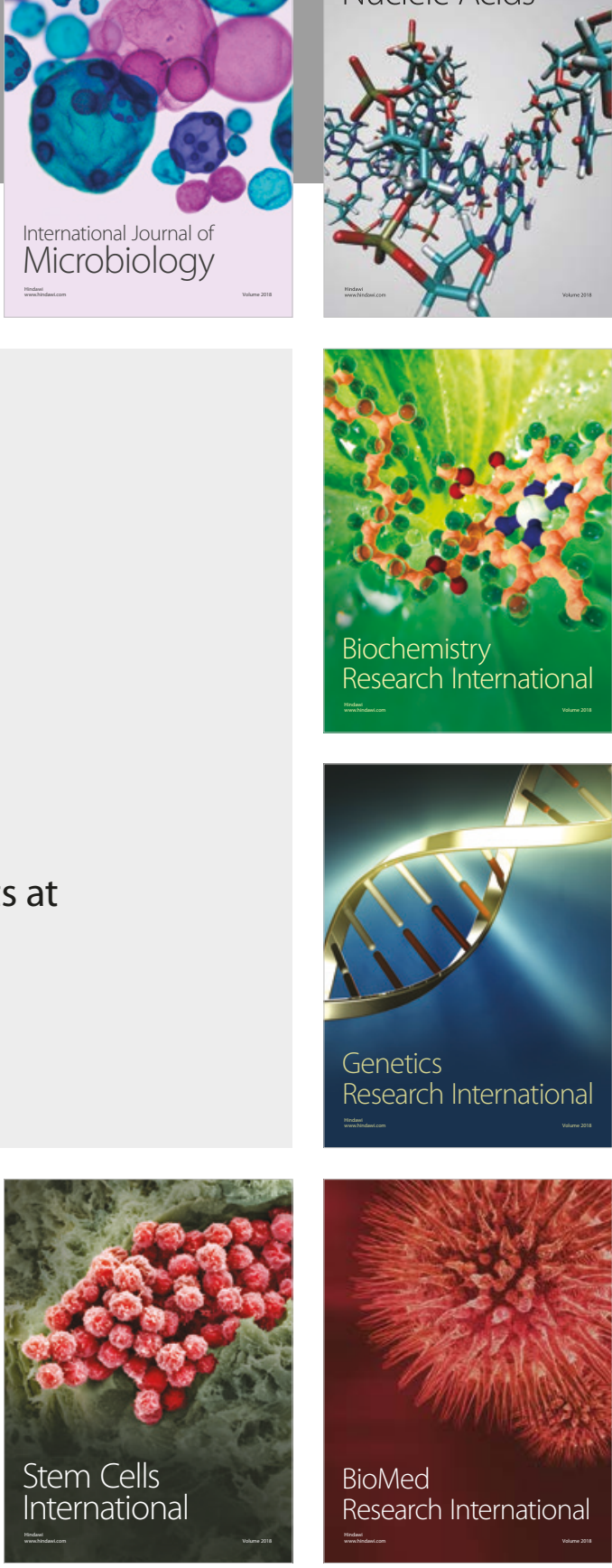
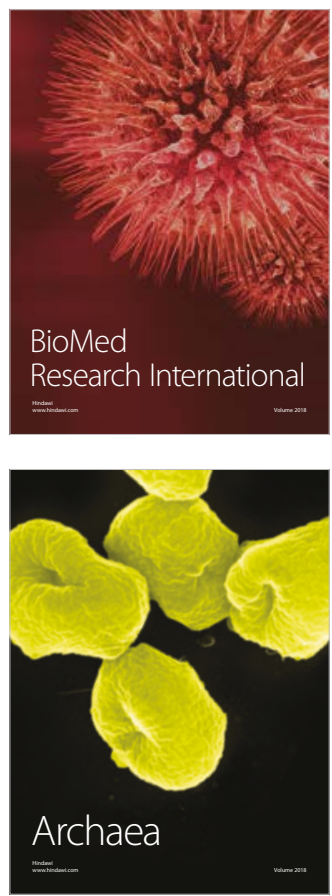